\title{
A Case of Expiratory Limb Filter Obstruction in Heated Circuit Kit
}

\author{
Misoon Lee, Eun Young Ko, So Jeong Lee, Ana Cho, Yang Hoon Chung, Bon Sung Koo, Joon-Ho Lee \\ Department of Anesthesiology and Pain Medicine, Soonchunhyang University Bucheon Hospital, Bucheon, Korea
}

\begin{abstract}
Antimicrobial filters that prevent cross-contamination through anesthesia equipment are commonly used in operating rooms. Occlusion of this filter leads to the patient's airway obstruction, which may lead to fatal outcomes. We report a case of the airway obstruction by antimicrobial filter occlusion during general anesthesia, and symptoms recovered immediately after removal of the filter.

Keywords: Airway obstruction; Filters; Equipment; Complications
\end{abstract}

\section{INTRODUCTION}

Several cases of cross-contamination of bacteria and viruses through anesthesia equipment have been reported [1,2]. To prevent this risk, an antibacterial filter is mounted on the breathing circuit to filter out microorganisms. It has been reported that there is a risk of airway obstruction by patient secretions [3] and mechanical damage [4], and there is still the possibility of an unexpected complications. Here, we reported a case of expiratory limb filter obstruction in a heated circuit kit.

\section{CASE REPORT}

A 51-year-old male (height $182 \mathrm{~cm}$, weight $88 \mathrm{~kg}$ ) patient was scheduled for modified radical neck dissection and free flap coverage on oropharyngeal cancer with neck metastasis. He underwent radiation therapy 6 months ago. Preoperative laboratory and image tests showed no abnormal results, but electrocardiogram showed left ventricular hypertrophy.

The patient was premedicated with glycopyrrolate $0.2 \mathrm{mg}$ intramuscular in the ward. On arrival of the patient to the operation room, non-invasive blood pressure monitoring, three leads electrocardiogram, pulse oximetry, and bispectral index measurement were performed in the operating room. After sufficient preoxygenation, general anesthesia was induced with intravenous adminis- tration of lidocaine (40 mg), propofol (150 $\mathrm{mg}$ ), and rocuronium $(50 \mathrm{mg})$. After uneventful anesthetic induction, anesthesia was maintained with continuous infusion of $2 \%$ propofol, remifentanil, and rocuronium. A central venous catheter was inserted into the left femoral vein and the left femoral artery was cannulated for blood pressure monitoring and arterial blood gas analysis. Before the surgical procedure, the otolaryngologist removed the endotracheal tube and performed a tracheostomy. Consequently, an endotracheal tube (internal diameter, $8.0 \mathrm{~mm}$ ) was inserted into the tracheostomy site for mechanical ventilation. During the operation, peak airway pressure was $13-14 \mathrm{~mm} \mathrm{Hg}$ with about $500 \mathrm{~mL}$ of tidal volume and $10-12 / \mathrm{min}$ of respiratory rate. We used the anesthetic machine (Datex-Ohmeda; GE Healthcare, Chicago, IL, USA) with the heated circuit kit (Mega Acer kit; ACE Medical, Seoul, Korea). The heated circuit kit consists of inspiratory limb that makes the anesthetic gases humidifier and heated by integrated heated wire and expiratory limb. Antimicrobial filters (MF filter, ACE Medical) with antibacterial, antiviral, and dehumidifying functions are mounted between the end of the expiratory tube and carbon dioxide absorbent.

About 12 hours after the operation, peak inspiratory pressure abruptly increased to over $25 \mathrm{~mm} \mathrm{Hg}$ from $12 \mathrm{~mm} \mathrm{Hg}$, and positive end-expiratory pressure (PEEP) to $18 \mathrm{~mm} \mathrm{Hg}$ from $4 \mathrm{~mm} \mathrm{Hg}$. We immediately stopped the operation, checked the patency of the tracheostomy tube, and suctioned to remove secretion. However, 
since no secretion was suctioned, we concluded that the obstruction of the tube due to the secretion was not the cause of the increase in airway pressure. With manual ventilation, we rechecked the whole circuit system and anesthetic workstation. Next, we suspected a problem with the breathing circuit and decided to remove the filter to rule out the possibility of sudden occlusion of the filter. Immediately after the filter was removed from the breathing circuit, airway resistance decreased dramatically, and PEEP and peak inspiratory pressure were also recovered to normal range. The total event time was about 20 minutes, and the vital sign was stable. Until the end of the surgery, the peak airway pressure maintained the range of 8 to $10 \mathrm{~mm} \mathrm{Hg}$ with $450 \mathrm{~mL}$ of tidal volume and $12 / \mathrm{min}$ of respiratory rate. The surgery resumed and ended after 4 hours. At the completion of the surgery, the patient was transferred to the intensive care unit with tracheostomy and was sedated to preserve the operation wound for 10 days. The patient was discharged 27 days after surgery without any respiratory complications.

\section{DISCUSSION}

For several years, it has been reported that the patient crosscontaminations through anesthetic equipment $[1,2]$. Therefore, in these days, it is common to apply antibacterial/antiviral filters to the breathing circuits to prevent cross-contaminations through anesthesia equipment.

However, the use of antimicrobial filters can cause unexpected complications, which can lead to fatal outcomes for the patient. In two previously reported cases of tension pneumothorax in patients due to obstruction of the antimicrobial filter, both of which were due to patient's secretions [3,4]. In addition, airway obstruction by sudden filter occlusion shows an increase in expiratory flow pressure $[5,6]$. This clinical manifestation is similar to bronchospasm. Therefore, anesthesiologists need careful monitoring, sufficient knowledge to appropriate and prompt response.

In this case, we were able to immediately detect increased peck inspiratory pressure and PEEP due to anesthetic workstation and ventilator alarms. We suspected the obstruction of the tube due to the patient's secretions but concluded that after the suction, the patency of the tube was maintained. Although the patient's vital signs were stable, ventilation was restored immediately after removal of the filter, and the patient was discharged without specific respiratory system complications after surgery, but the risk of the obstruction of the filter during general anesthesia should never be overlooked.

We have not done a histological examination of the removed filter. Therefore, the exact reason for the filter occlusion is still unknown. However, two possibilities can be assumed. First, this may form and absorb excess water within the filter. Schummer et al. [7] has reported a filter blockage for the same reason. While the manufacturer's recommended usage time is 4 hours but at that time, we have been using the heated circuit kit for over 12 hours. The prolonged use of the antimicrobial filter caused the formation of invisible water inside the breathing circuit which accumulated in the filter and eventually blocked the filter, causing PEEP and reducing airway compliance. Second, the patient's sputum may also be cause of filter occlusion. In the several previous studies, occlusion caused by secretions such as sputum [3] and edema fluid [8] has been reported. In conclusion, airway obstruction with filter occlusion may lead to fatal outcomes and the possibility of this problem should be considered when sudden airway pressure or PEEP increases during mechanical ventilation.

\section{REFERENCES}

1. Olds JW, Kisch AL, Eberle BJ, Wilson JN. Pseudomonas aeruginosa respiratory tract infection acquired from a contaminated anesthesia machine. Am Rev Respir Dis 1972;105:628-32.

2. Chant K, Kociuba K, Munro R, Crone S, Kerridge R, Quin J, et al. Investigation of possible patient-to-patient transmission of hepatitis $\mathrm{C}$ in a hospital. N S W Public Health Bull 1994;5:47-51.

3. McEwan AI, Dowell L, Karis JH. Bilateral tension pneumothorax caused by a blocked bacterial filter in an anesthesia breathing circuit. Anesth Analg 1993;76:440-2.

4. Smith CE, Otworth JR, Kaluszyk P. Bilateral tension pneumothorax due to a defective anesthesia breathing circuit filter. J Clin Anesth 1991;3:229-34.

5. Aarhus D, Soreide E, Holst-Larsen H. Mechanical obstruction in the anaesthesia delivery-system mimicking severe bronchospasm. Anaesthesia 1997;52:992-4.

6. Chung MY, Yang WJ, Lee JY. Airway obstruction in heat \& moisture exchanger filter: a case report. Anesth Pain Med 2011;6:96-9.

7. Schummer W, Schummer C, Fuchs J, Voigt R. Sudden upper airway obstruction due to invisible rain-out in the heat and moisture exchange filter. Br J Anaesth 2002;89:335-6.

8. Kopman AF, Glaser L. Obstruction of bacterial filters by edema fluid. Anesthesiology 1976;44:169-70. 